\title{
Brain Tumor Detection, Classification, Tracking and 3D Modelling
}

\author{
Aman Doshi \\ Department of Computer \\ Engineering \\ KJ Somaiya College of \\ Engineering \\ Mumbai, India
}

\author{
Miloni Shash \\ Department of Computer \\ Engineering \\ KJ Somaiya College of \\ Engineering \\ Mumbai, India
}

\author{
Sonia Patel \\ Department of Computer \\ Engineering \\ KJ Somaiya College of \\ Engineering \\ Mumbai, India
}

\author{
Zaheed Shaikh \\ Department of Computer Engineering \\ KJ Somaiya College of Engineering \\ Mumbai, India
}

\begin{abstract}
A vital role is impersonated by medical imaging within the field of medication. Image segmentation is a popularly identified technique that is employed to extricate unnatural tissues in medical images. There is a chance of a tumor getting converted into lethal cancer which is a significant leading cause of loss of life. This can be detected accurately by using magnetic resonance imaging images(MRI) in an automatic system. It will also consume less time in detecting the tumor.
\end{abstract}

Automating the entire method of detection the tumor at an initial stage helps to cure it quicker. The proposed work includes using magnetic resonance(MRI) images which will be enhanced by performing the mentioned Pre-Processing techniques like thresholding, watershed and other morphological operations which would help us in identifying and detecting the tumor. The features would be extracted and a delta graph will be generated which will help us in creating a $3 \mathrm{D}$ model of the brain. The 3D model helps the doctors to make a decision which kind of cure operation is to be performed and therefore gain a deeper insight. The proposed work will also classify the detected tumor into Begin or Malignant with the help of K-means and Artificial Neural Network. Also, the growth of the brain tumor will be tracked with the help of differential analysis. The projected technique will be implemented with the aid of MATLAB R2018b.

\section{Keywords}

K-means, Morphological operator

\section{INTRODUCTION}

The brain is the most essential part of our body. All the functions within the body are regulated by the brain. In order, to digitally visualize the inner structure of the brain MRI and CT scan pictures are used. within the MRI scan, the extraction of sentimental tissues and therefore the internal structure of the brain happens. A distinction between traditional and abnormal tissues is quite visible. MRI uses magnetic radio waves to create the details of the internal organ. As there's no radiation emitted, MRI is widely accepted everywhere the globe. The tumor is outlined as the growth of the abnormal cell. A lump of tissues therefore formed is known as to a tumor.

The planned methodology takes z-stack MRI images as an input. Pre-Processing steps are performed to enhance the images and noise removal.The proposed methodology will perform the Feature Extraction and Delta Graph which will help in identifying the tumor.Following the Delta graph,detection of the tumor will be performed which will help to detect the presence of a tumor in the brain. The 3D model of the brain and the detected tumor will be created for a deeper insight for diagnosing. After the 3D model being created, the classifier will classify the tumor as Benign or Malignant. The last step will track the growth that is the size of the tumor.

\section{LITERATURE SURVEY}

Pratomo Adhi Nugroho, Dwi Kurnia Basuki \& Riyanto Sigit[1] have proposed a technique to convert $2 \mathrm{D}$ image to $3 \mathrm{D}$ image using the Marching Cubes algorithm. Many 2D images are clicked and passed through filtering. In filtering, noise is removed from the image and the resultant image is a more sharpened image. After filtering, the marching cubes algorithm is used to recreate the surface of the object.

Sharma M., Purohit G.N \& Mukherjee S[3] have proposed a method which uses a hybrid technique for extraction and detection of the tumor from MRI image. The required features were extracted using, Grey Level Co-occurrence Matrix (GLCM) technique. In order to detect the tumor, K-means and artificial neural network (KMANN) techniques are used. Kmeans clustering partitions the data into $\mathrm{k}$ groups of data. There are two phases wherein, it calculates the $\mathrm{k}$ centroid and then selection of nearest centroid from the respective data point. Using the extracted features fuzzy rules are created for a neurofuzzy inference system.

Z. N. K. Swati, Q. Zhao, M. Kabir, F. Ali, A. Zakir, S. Ahmad, and J. metal [4] has projected an associate automatic contentbased image retrieval (CBIR) system for brain tumors on T1weighted contrast-enhanced magnetic resonance images (CEMRI). The paper represents tumor feature extraction using deep learning. Deep learning can depict low and high-level data fully and combined the self-learning phase in feature extraction.

G. Manogaran, P. M. Shakeel, A. S. Hassanein, P. M. Kumar, and G. C. Babu[5] have projected the associate degree improved orthogonal gamma distribution-based machinelearning the approach is employed to analyze the undersegments and over-segments of brain tumor regions to mechanically sight abnormalities inside the ROI( region of interest). Any data imbalances as a result of improper edge 
matching inside the abnormal region are sampled by matching the string coordinates and sensitivity, and additionally, the desired parameters are measured and extracted using machine learning.

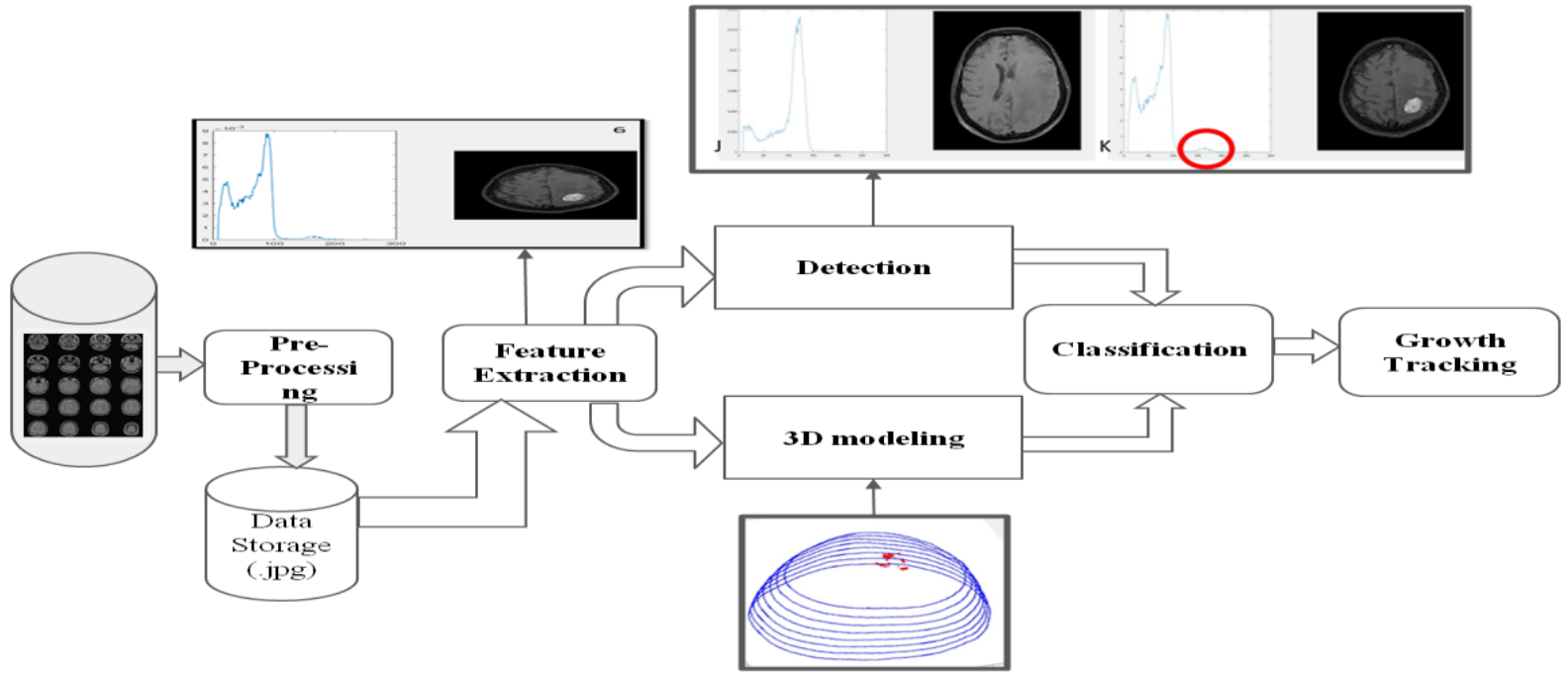

Fig. 1: System Architecture

In this study[6], brain tumor datasets are considered for analysis. Rotation invariance technique is implemented to extract the native binary pattern features of the tumor. Due to this the tumor shifts and rotational changes occur. The brain image remains stationary corresponding to the coordinate system. This gives detailed results of the texture features of hidden layers of the tumor. The structural framework of the neural network is constructed based on the extracted feature. To enhance image feature extraction multi-channel input for the neural network is also developed. Convolution rules of Xception and Dense net are developed to increase efficiency. Test result testifies the efficiency of the neural network.

\section{PROPOSED METHODOLOGY}

In the proposed methodology, the dataset comprises of various zstack images in the form of MRI image. The suggested method is able to classify the recognized tumor into Benign and
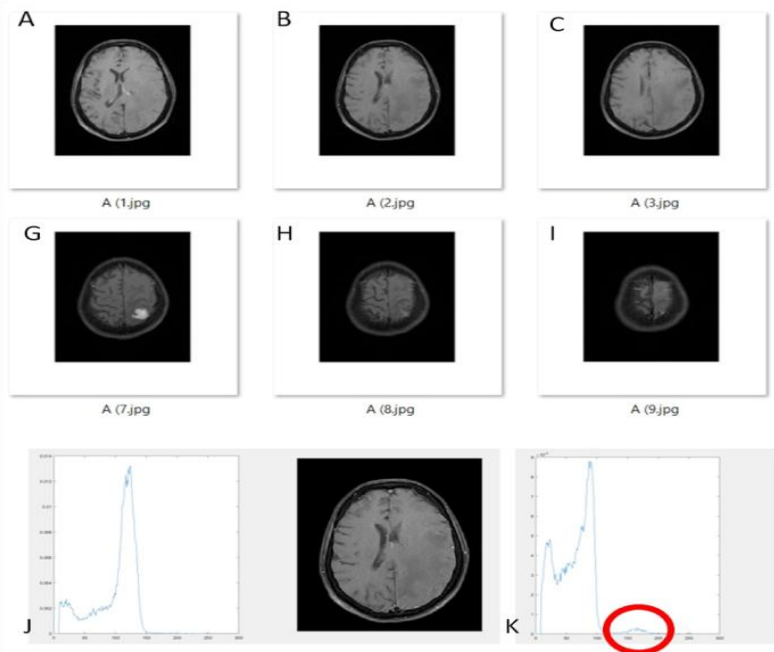

Malignant tumor. The most tedious task in tumor classification and segmentation is to identify the Malignant tumor. Examining all the varied features of the tumor increases the diagnosis rate. Generated noise is removed with help histogram stretching, pixel normalization, and thresholding. Several other PreProcessing techniques are used to help in accurately identifying, classifying and creating a 3D model of the brain. The recommended methodology creates a Delta graph which helps in the feature extraction process, which in turn helps in accurately identifying the tumor in the brain. The classification of the tumor will be done with the help of K-means and the neural networks. The 3D model of the brain will help the radiologists in correctly planning the treatment and gain a deeper insight into the structure of the brain. The growth and the size of the tumor will be calculated with the help of differential analysis. The output of the proposed methodology can be understood from the figure Fig.1 given above.

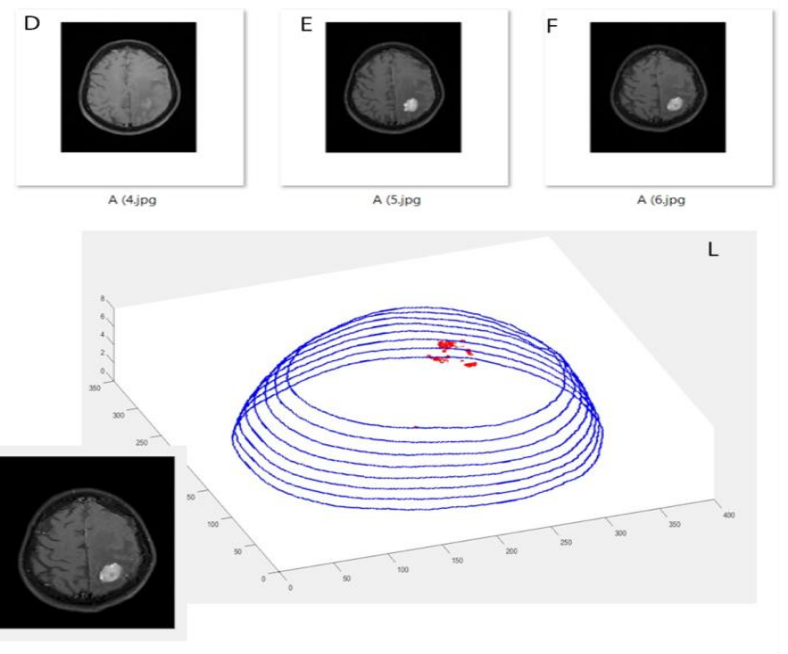

Fig 2: Detection of the Tumor and 3D View

The intended technique is implemented using the data set of Zstack images. The output of the detected tumor, the proximity of the tumor to the periphery of the brain plays a substantial role in analyzing the tumor type and treatment selection. The stated method renders better outcomes on tumor classification, 3d modeling and growth tracking of various z-stack images of the brain. The Fig.3 below illustrates the results of the proposed methods tested on the z-stack magnetic resonance imaging images. Fig. 3 (A1 to A5) are the input magnetic resonance imaging images, the proposed technique advances the identification results, decreases error, enhances the efficiency and lessens the false detection wherein image A1 is the output 
of the cropped image, A2 represents morphological operations, $\mathrm{A} 3$ represents the output of the brain after edge detection ,A4 is the output image of the technique called Principle Component Analysis (PCA) and (A5) displays the output of the watershed algorithm . Fig.2 ( $\mathrm{J}$ and $\mathrm{K}$ ) shows the delta graph of the brain and therefore the detected tumor. The recommended methodology is tested on both regular and irregular tumor images. The problem of multiple tumor classification and erroneous detection is resolved using the proposed segmentation. In Fig.2(J) the absence of the bump indicates there is no tumor, whereas within the Fig. $2(\mathrm{~K})$ the red circled part shows the bump that indicates the presence of the tumor. The proposed technique shows the naive 3D model of the brain and also the detected tumor in Fig.2(L) where the blue color indicates the z-slice if the brain and the red color represents the detected tumor. The recommended techniques help the doctors and experts to accurately detect tumor and classify it.
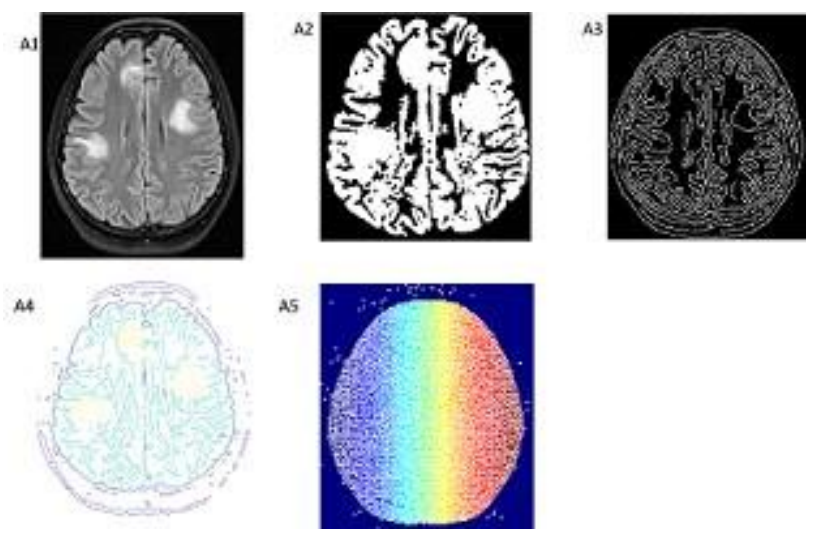

Fig 3: Preprocessing Techniques

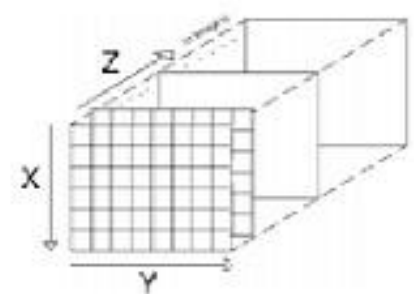

Fig 4: 3D Modeling

\subsection{3-Dimensional model of the brain}

This section discusses how the $3 \mathrm{D}$ model of the brain is constructed using the z-stack images which comprise of tumor cells. The result demonstrates that the technique used is quite accurate and creates a 3D model in a very short period of time. The segmented MRI images are pushed one by one to form a 3D cube-like structure. This helps the surgeons in gaining a deeper insight into the structure of the brain and also aids the surgeon to get a proper judgment for the operation to be performed. 3D view depends on the accurate location and volume of the tumor. The purpose is to improve the view of the detected tumor by stacking the $2 \mathrm{~d}$ image[2], keeping it with the $\mathrm{z}$-axis, thereby improving the quantitative properties of the image. Every 2D matrix fa(x,y) is put on the top of every image to create a 3-dimensional model. The proposed method appears in Fig. 4. They are primarily 2D matrices stacked to form 3D matrix.

\subsection{Classifier Output}

Classification results of two significant types of tumor are shown in Fig.5. The results certainly, confirms that the recommended approach can classify the tumor with diminished error and great efficiency. Localization of the tumor helps in the classification of a tumor that is Benign or Malignant. The output in the Fig.5 shows the accuracy achieved with the help of Confusion Matrix(A1), Prediction Chart(A2)and ROC Curve (A3).

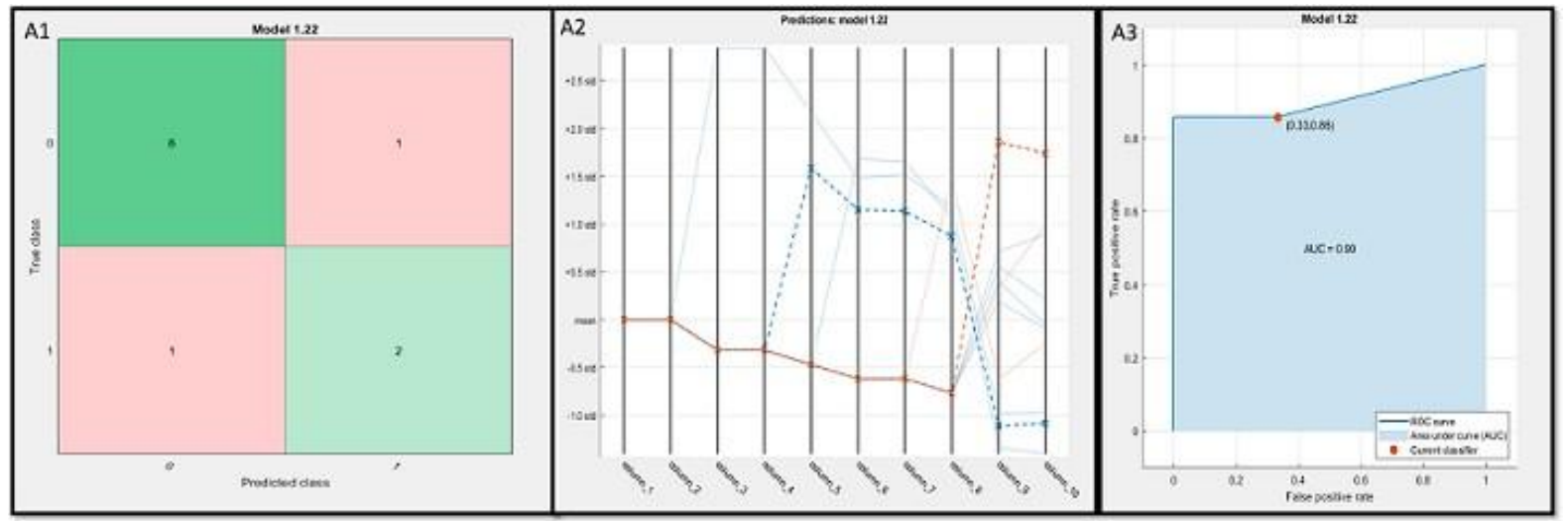

Fig 5: Different Charts of The Classifier Output

\subsection{Growth Tracking}

The Fig. 6 shows the output of the size of the tumor increased or decreased over a period of time.Fig.6(A1) represents the original input MRI image and (A2) represents an increased size of the tumor. The same is the case with the output B1 where (B1) represents the original input MRI image of the tumor and (B2) shows that there is a significant growth reduction in the size of the tumor. This method is implemented on the data sets of two different patients. 

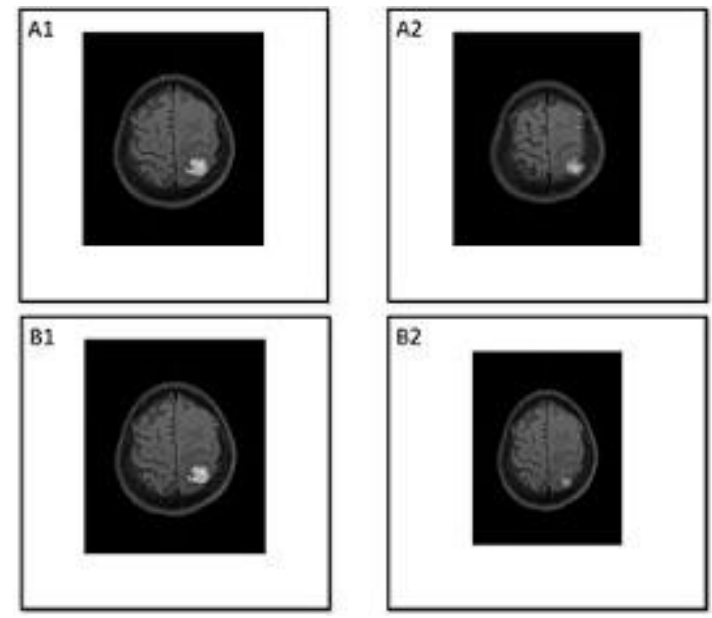

Fig 6: Growth Tracking of The Tumor

\section{CONCLUSION}

This paper uses a completely automated approach for classifying, detecting and identifying the growth of the brain tumor. The proposed approach also helps to recreate the 3D model, which helps the doctor to perform a mock operation. Due to this, the success rate of the operation can be increased. The proposed method uses MRI images as an input image because MRI image is a standard image for detecting brain tumor by the doctors.Elimination of the PreProcessing step will not generate accurate results.

\section{FUTURE WORK}

The 3D model can be modified and can be used to capture deeper insight of the brain by providing functions like zoom in, zoom out, editing, etc. It can also be trained to suggest the successful medicines, treatment or surgery for the detected tumor. The stage or level of the tumor can also be determined with the voluminous dataset. Along with the classification of tumor, the different types of the tumor names to be displayed. The survival chances can also be predicated with the well trained neural network.

\section{ACKNOWLEDGMENT}

Thanking Dr.Madhura Mehendale to guide and provide us with the data set of the brain tumor.

\section{REFERENCES}

[1] P. A. Nugroho, D. K. Basuki, and R. Sigit, 3D heart image reconstruction and visualization with marching cubes algorithm, 2016 International Conference on Knowledge Creation and Intelligent Computing (KCIC), 2016.

[2] S. Roy, D. Bhattacharyya, S. K. Bandyopadhyay, and T.H. Kim, An improved brain MR image binarization method as a preprocessing for abnormality detection and features extraction, Frontiers of Computer Science, vol. 11, no. 4, pp. 717727, 2018.

[3] M. Sharma, G. N. Purohit, and S. Mukherjee, Information Retrieve from Brain MRI Images for Tumor Detection Using Hybrid Technique K-means and Artificial Neural Network (KMANN), Networking Communication and Data Knowledge Engineering
Lecture Notes on Data Engineering and Communications Technologies, pp. 145157, Mar. 2018.

[4] Z. N. K. Swati, Q. Zhao, M. Kabir, F. Ali, A. Zakir, S. Ahmad, and J. Lu, Content-Based Brain Tumor Retrieval for MR Images Using Transfer Learning, IEEE Access, pp. 11, 2019.

[5] G. Manogaran, P. M. Shakeel, A. S. Hassanein, P. M. Kumar, and G. C. Babu, Machine Learning ApproachBased Gamma Distribution for Brain Tumor Detection and Data Sample Imbalance Analysis, IEEE Access, vol. 7, pp. 1219, 2019.

[6] A.-M. Yang, X.-L. Yang, W.-R. Wu, H.-X. Liu, and Y.$X$. Zhuansun, Research on Feature Extraction of Tumor Image Based on Convolutional Neural Network, IEEE Access, pp. 11, 2019.

[7] S. Goswami and L. K. P. Bhaiya, A hybrid neuro-fuzzy approach for brain abnormality detection using GLCM based feature extraction, 2013 International Conference on Emerging Trends in Communication, Control, Signal Processing and Computing Applications (C2SPCA), 2013.

[8] Y. Megersa and G. Alemu, Brain tumor detection and segmentation using hybrid intelligent algorithms, Africon 2015, 2015.

[9] Amin, Safaa Elsayed, and M. A. Megeed, Brain tumor diagnosis systems based on artificial neural networks and segmentation using MRI. 2012 8th International Conference on Informatics and Systems (INFOS) (2012): MM-119-MM-124.

[10] M. Masroor Ahmed \& Dzulkifli Bin Mohammad(2010), "Segmentation of Brain MRI Images for Tumor Extraction by Combining K-means Clustering and Perona-Malik Anisotropic Diffusion Model," International Journal of Image Processing, Volume (2): Issue(I) 27.

[11] E. Abdel-Maksoud, M. Elmogy, and R. Al-Awadi, Brain tumor segmentation based on a hybrid clustering technique, Egyptian Informatics Journal, vol. 16, no. 1, pp. 7181, 2015.

[12] E. F. Badran, E. G. Mahmoud, and N. Hamdy, An algorithm for detecting brain tumors in MRI images, The 2010 International Conference on Computer Engineering \& Systems, 2010.

[13] R. Banik, M. R. Hasan, and M. S. Iftekhar, Automatic detection, extraction and mapping of Brain Tumor from MRI scanned images using Frequency Emphasis Homomorphic and cascaded hybrid Filtering techniques, 2015 International Conference on Electrical Engineering and Information.

[14] H. S. Abdulbaqi, M. Z. M. Jafri, K. N. Mutter, A. F. Omar, I. S. Mustafa, L. K. Abood, "Segmentation and Estimation of Brain Tumor Volume in Magnetic Resonance Images Based on T2-Weighted using Hidden Markov Random Field Algorithm", Journal of Telecommunication Electronic and Computer Engineering (JTEC), vol. 8, pp. 9-13, 2016. 